\title{
Hard discs under steady shear: comparison of Brownian dynamics simulations and Mode Coupling Theory
}

\author{
By Oliver Henrich ${ }^{1}$, Fabian Weysser ${ }^{2}$, Michael E. Cates ${ }^{1}$, Matthias \\ FuCHS $^{2}$ \\ ${ }^{1}$ School of Physics and Astronomy, University of Edinburgh, JCMB, The King's \\ Buildings, Edinburgh EH9 3JZ, UK \\ ${ }^{2}$ Universität Konstanz, Fachbereich Physik, D-78457 Konstanz, Germany
}

\begin{abstract}
Brownian dynamics simulations of bidisperse hard discs moving in two dimensions in a given steady and homogeneous shear flow are presented close to and above the glass transition density. The stationary structure functions and stresses of shear melted glass are compared quantitatively to parameter free numerical calculations for monodisperse hard discs using mode coupling theory (MCT) within the integration through transients (ITT) framework. Theory qualitatively explains the properties of the yielding glass but quantitatively overestimates the shear driven stresses and structural anisotropies.
\end{abstract}

\section{Keywords:}

\section{Introduction}

Shear flow can drive dense colloidal dispersions into states far from equilibrium. Especially of interest is the possibility to shear melt colloidal solids, in particular metastable colloidal glasses and gels, and to investigate shear-melted (yielding) colloidal glasses. Does a yield stress and/or yield strain exist (Petekidis et al. 2002)? Are shear-melted states necessarily heterogeneous (e.g. shear banded)? Does ageing prevent stationary states under steady shearing? Do hydrodynamic interactions dominate the properties in flow? These are just some of the questions whose answers will provide insights into the still murky glassy state.

Recently the integration through transients (ITT) approach has been used to generalize the mode coupling theory (MCT) of the structural glass transition to the case of steady shearing (Fuchs Cates 2002, 2009), and to arbitrary homogeneous flows (Brader et al. 2008), albeit based on a number of approximations. First, homogeneous flow profiles are assumed from the outset, second, hydrodynamic interactions are neglected, and third, the mode coupling (mean-field-like) decoupling approximation, splitting a four point density fluctuation function into the product of two-point density correlators, has been applied. While simplified (schematic) models of MCT-ITT (Fuchs Cates 2003) can well be fitted to the linear rheology (frequency dependent storage and loss moduli) and non-linear rheology (flow curves, viz. stress as function of shear rate) of model dispersions (Siebenbürger et al. 2009), no fully quantitative comparison between the theory and data without additional approximations has been presented up to now. 
We provide this first quantitative comparison, solving the MCT-ITT equations for hard discs confined to move in a plane, and performing Brownian dynamics simulations for a binary hard disc mixture, also in two dimensions. Computational (memory) constraints force us to work in two dimensions, which is still of some experimental relevance, as glasses have been observed in quasi-two-dimensional dispersions (Bayer et al. 2007). Use of a binary mixture suppresses the nucleation rate sufficiently for even our long simulation runs to remain free of crystal nuclei. As hydrodynamic interactions are absent also in the simulation, and as Lees-Edwards boundary conditions (LE) in combination with the thermostat impose a homogeneous shear rate, the integration of the equations of motion in the simulation ensure a stringent test of the theory. Results for the stationary shear and normal stresses, and for the shear-distorted microstructure are presented and discussed. The input quantities entering the theory, and transient density correlation functions, which (crucially) enter during intermediate MCT-ITT steps, are characterized also.

Our work bears some similarity to the study by Miyazaki, Reichman, and Yamamoto, who, however, concentrated on fluid states under shear, and on their time dependent fluctuations (Miyazaki et al. 2004). We focus here on the yielding glass state, and its stationary, time-independent averages, which are not accessible to the theory in Ref. (Miyazaki et al. 2004). In order to compare quantitatively with theory, we aimed for better statistics than in comparable previous two-dimensional simulation studies of sheared glasses (Yamamoto Onuki 1998; Furukawa et al. 2009).

\section{Simulation}

The basic concept of the algorithm has been described in detail in three dimensions in (Scala et al. 2007) and can easily be adapted to two dimensions $x$ and $y$. In order to prevent crystallisation at high densities we consider a binary mixture with a diameter ratio of $D_{2} / D_{1}=1.4$ at equal number concentrations $\left(N_{\text {small }}=N_{\text {big }}\right)$. $N=1000$ hard discs move in a simulation box of volume $V$ with periodic Lees Edwards boundary conditions at packing fraction $\varphi=\frac{N \pi}{8 V} \cdot\left(D_{1}^{2}+D_{2}^{2}\right)$. The mass of the particles is set equal to unity, thermal energy $k_{B} T$ sets the energy scale, and $D_{1} \equiv D$ is used as unit of length in the following. We choose our coordinate axes such that flow is in the $x$-direction and the shear gradient $\dot{\gamma}$ is in the $y$-direction. After putting the particles on their initial positions we provide Gaussian distributed velocities which will be overlain by the linear shear flow. To propagate the system forwards in time we employ a semi-event-driven algorithm. For every particle at the time $t_{0}$ the algorithm determines the possible collision time $\tau$ with any other particle. This is easily achieved by solving the equation

$$
\frac{1}{2}\left(D_{i}+D_{j}\right)=\left|\boldsymbol{r}_{i j}-\boldsymbol{v}_{i j} \tau\right|
$$

where $D_{i}$ and $D_{j}$ denote the diameters of the particles $i$ and $j, \boldsymbol{r}_{i j}$ the relative vector between both particles, and $\boldsymbol{v}_{i j}$ the relative velocities. The smallest solution $\tau_{m i n}$ for all particle pairs determines the next event in the algorithm. All particles can then be propagated according to: $\boldsymbol{r}\left(\tau_{\min }\right)=\boldsymbol{r}\left(t_{0}\right)+\boldsymbol{v} \tau_{\min }$. With the conservation of energy and momentum the binary collision laws impose new velocities $\boldsymbol{v}_{j}^{*}$ for the particle $j$ and $i$

$$
\boldsymbol{v}_{j}^{*}=\boldsymbol{v}_{j}+\left(\hat{\boldsymbol{r}}_{i j} \cdot \boldsymbol{v}_{i j}\right) \hat{\boldsymbol{r}}_{i j}, \quad \boldsymbol{v}_{j}^{*}=\boldsymbol{v}_{j}-\left(\hat{\boldsymbol{r}}_{i j} \cdot \boldsymbol{v}_{i j}\right) \hat{\boldsymbol{r}}_{i j} .
$$


Due to the boundary conditions any particle in the vicinity of the box-boundary can collide with an image particle coming from the other end of the box. The boxes are constructed in such a way that they are translated with the velocity $\dot{\gamma} L$, where $L$ denotes the size of the box. The boundary conditions thus have the consequence that the velocities tend to increase. Therefore a thermostat has to be introduced. After a time $\tau_{B}=0.01$ a so-called Brownian step sets in, which assures that the particles move diffusively for longer times. In the Brownian step at the time $\tau_{B}$, all particle velocities are freshly drawn from a Gaussian distribution with $\left\langle|\boldsymbol{v}|^{2}\right\rangle=2$. After that linear shear flow is imposed so that $\langle\boldsymbol{v}\rangle=\dot{\gamma} y\left(\tau_{B}\right) \hat{\mathbf{x}}$ holds.

As the system starts from a cubic lattice it is necessary to wait for the system to relax before meaningful stationary averages can be taken. The quantity of interest in the work presented here is the potential part of the stress: $\sigma_{\alpha \beta}(\dot{\gamma})=$ $\frac{1}{V}\left\langle\sum_{i j}\left(\boldsymbol{F}(t)_{i j}\right)_{\alpha}\left(\boldsymbol{r}(t)_{i j}\right)_{\beta}\right\rangle^{(\dot{\gamma})}$, with the relative force components (in $\alpha$-direction) of particles $i$ and $j\left(\boldsymbol{F}(t)_{i j}\right)_{\alpha}$, and the particles' relative distance components $\left(\boldsymbol{r}(t)_{i j}\right)_{\beta}$. The kinetic part will play no role, and thus has already been omitted. As we consider hard particles the forces must be calculated from the collision events. By observing the collisions within a certain time window $\Delta \tau_{c}$ forces may be extracted using the change of momentum which occurs during the observation time. This leads to the evaluation algorithm (Lange et al. 2009; Foss Brady 2000)

$$
\sigma_{\alpha \beta}(\dot{\gamma})=\left\langle\frac{1}{\Delta \tau_{c}} \sum_{t_{c} \in\left\{t, t+\Delta \tau_{c}\right\}}\left(\Delta \boldsymbol{v}\left(t_{c}\right)_{i j}\right)_{\alpha}\left(\boldsymbol{r}\left(t_{c}\right)_{i j}\right)_{\beta}\right\rangle_{s},
$$

where the summation is over all collisions of particles $i$ and $j$ at time $t_{c}$ within the time window $\Delta \tau_{c}$. The procedure effectively sums the momentum changes $\left(\Delta \boldsymbol{v}_{i j}\right)_{\alpha}$ in the $\alpha$ - direction multiplied by the relative distance of the particles $\left(\boldsymbol{r}_{i j}\right)_{\beta}$ in the $\beta$ - direction. Here and below the brackets $\langle\ldots\rangle_{s}$ denote the average over different simulation runs.

Additionally the shear stress can be computed via the contact value $g(\boldsymbol{r})$ :

$$
\sigma_{x y}(\dot{\gamma})=\frac{8 k_{B} T}{\pi^{2}} \varphi^{2} \sum_{i, j \in\{1,2\}} \frac{1}{d_{i j}^{2}} \int \mathrm{d} \theta \cos (\theta) \sin (\theta) g^{i j}(r, \theta)
$$

where $g^{11}(r, \theta), g^{12}(r, \theta) \ldots$ denote the $\theta$-dependent partial contact values of the two components and $d_{11}, d_{12}, \ldots$ the minimal distance between two particles. $\theta$ is the polar angle.

The Green-Kubo relation $\eta_{x y}=\frac{1}{k_{B} T V} \int_{0}^{\infty}\left\langle\sigma_{x y}(t) \sigma_{x y}(t+s)\right\rangle \mathrm{d} s$ holds for the nonsheared system. Thus the shear viscosity can be extracted from the simulation via (Alder et al. 1970)

$$
\eta_{x y}=\frac{1}{2 k_{B} T V} \lim _{t \rightarrow \infty} \frac{1}{t}\left\langle\left(\int_{0}^{\infty} \sigma_{x y}\left(t^{\prime}\right) \mathrm{d} t^{\prime}\right)^{2}\right\rangle=\frac{1}{V}\left\langle\left(\sum_{\text {coll }} \boldsymbol{r}_{y} \Delta \boldsymbol{v}_{x}\right)^{2}\right\rangle_{s}
$$

where the sum runs over all collisions. The second pivot in this analysis is the equaltime structure factor and its deviation from the quiescent system. Exploiting that 


$$
\begin{gathered}
S_{\boldsymbol{q}}(\dot{\gamma})=\left\langle\frac{1}{N} \sum_{i, j} \exp \left(i \boldsymbol{q}\left(\boldsymbol{r}_{i}-\boldsymbol{r}_{j}\right)\right)\right\rangle^{(\dot{\gamma})} \text { is a real quantity, we can extract it via } \\
S_{\boldsymbol{q}}(\dot{\gamma})=\left\langle\frac{1}{N} \sum_{(i, j) \in N I} \cos \left(\boldsymbol{q}\left(\boldsymbol{r}_{i}-\boldsymbol{r}_{j}\right)\right)\right\rangle_{s}
\end{gathered}
$$

where the double sum runs over all pairs of particles $i$ and $j(N I)$. The pairs $i j$ are determined by the LE boundary conditions and the constraint of having the lowest distance among all possible image particles in the surrounding boxes.

For low Peclet numbers $\left(\mathrm{Pe}_{0}=\dot{\gamma} D^{2} / D_{0}\right)$ in fluid states, $g(\boldsymbol{r})$ can be expanded: $g(\boldsymbol{r})=$ $g_{0}(r)+2 \mathrm{Pe}_{0} \frac{x y}{r^{2}} g_{1}(r)+\mathcal{O}\left(\mathrm{Pe}_{0}^{2}\right)$. This result can be used to derive the relative distortion of the structure factor in the linear response regime (Strating 1999).

$$
\delta S(\boldsymbol{q}, \dot{\gamma} \rightarrow 0)=2 \mathrm{Pe}_{0} \int \mathrm{d} r r g_{1}(r) \int \mathrm{d} \theta \cos (\theta) \sin (\theta) \mathrm{e}^{\left[i\left(r q_{x} \cos (\theta)+r q_{y} \sin (\theta)\right)\right]}
$$

while $g_{1}(r)$ can be obtained via

$$
\frac{2 \pi}{\mathrm{Pe}_{0}} \int \mathrm{d} \theta g(\boldsymbol{r}) \cos (\theta) \sin (\theta)=g_{1}(r)+\mathcal{O}\left(\mathrm{Pe}_{0}^{2} \cdot x y / r^{2}\right) .
$$

\section{Mode Coupling Theory in the integrations through transients framework}

The MCT-ITT approach generalizes the MCT of the glass transition to colloidal dispersions under strong continuous shear. It considers $N$ spherical particles with arbitrary interaction potential which move by Brownian motion relative to a given linear shear profile. An equation of motion for a transient density correlator $\Phi_{\mathbf{q}}(t)$ encodes structural rearrangements, and approximated generalized Green-Kubo laws relate stress relaxation to the decay of density fluctuations.

The transient density correlator is defined by $\Phi_{\mathbf{q}}(t)=\left\langle\rho_{\mathbf{q}}^{*} \rho_{\mathbf{q}(t)}(t)\right\rangle / N S_{q}$, where the density fluctuation is as usual $\rho_{\mathbf{q}}(t)=\sum_{j=1}^{N} \exp \left\{i \mathbf{q} \cdot \mathbf{r}_{j}(t)\right\}$, and, in ITT, the average can be performed over the equilibrium Gibbs-Boltzmann ensemble. Thus the normalization of the initial value $\Phi_{\mathbf{q}}(0)=1$ is given by the equilibrium structure factor $S_{q}$. The time-dependent or shear-advected wavevector $\mathbf{q}(t)=\left(q_{x}, q_{y}-\dot{\gamma} t q_{x}\right)^{T}$ appearing in the definition eliminates the affine particle motion with the flow field, and gives $\Phi_{\mathbf{q}}(t) \equiv 1$ in the absence of Brownian motion. Shear flow coupled to random motion causes $\Phi_{\mathbf{q}}(t)$ to decay, as given by an (exact) equation of motion containing a retarded friction kernel which arises from the competition of particle caging and shear advection of fluctuations

$$
\dot{\Phi}_{\mathbf{q}}(t)+\Gamma_{\mathbf{q}}(t)\left\{\Phi_{\mathbf{q}}(t)+\int_{0}^{t} d t^{\prime} m_{\mathbf{q}}\left(t, t^{\prime}\right) \dot{\Phi}_{\mathbf{q}}\left(t^{\prime}\right)\right\}=0
$$

where the initial decay rate contains Taylor dispersion as $\Gamma_{\mathbf{q}}(t)=q^{2}(t) / S_{q(t)}$. The generalized friction kernel $m_{\mathbf{q}}\left(t, t^{\prime}\right)$, which is an autocorrelation function of fluctuating stresses, is approximated, following MCT precepts by an expression involving the structural rearrangements captured in the density correlators

$m_{\mathbf{q}}\left(t, t^{\prime}\right)=\int \frac{d^{2} k}{(2 \pi)^{2}} \frac{n S_{q(t)} S_{k\left(t^{\prime}\right)} S_{p\left(t^{\prime}\right)}}{2 q^{2}(t) q^{2}\left(t^{\prime}\right)} V_{\mathbf{q k p}}(t) V_{\mathbf{q} \mathbf{k p}}\left(t^{\prime}\right) \Phi_{\mathbf{k}\left(t^{\prime}\right)}\left(t-t^{\prime}\right) \Phi_{\mathbf{p}\left(t^{\prime}\right)}\left(t-t^{\prime}\right)(3$ 
with abbreviation $\mathbf{p}=\mathbf{q}-\mathbf{k}, n$ the particle density, and a vertex function given by

$$
V_{\mathbf{q k p}}(t)=\mathbf{q}(t) \cdot\left(\mathbf{k}(t) c_{k(t)}+\mathbf{p}(t) c_{p(t)}\right)
$$

where $c_{k}$ is the Ornstein-Zernicke direct correlation function $c_{k}=\left(1-1 / S_{k}\right) / n$. An additional memory kernel is neglected (Fuchs Cates 2009). The equilibrium structure factor, $S_{k}$, encodes the particle interactions and introduces the experimental control parameters like density and temperature. Similarly, the potential part of the stress $\sigma_{\alpha \beta}(\dot{\gamma})=\left\langle\sigma_{\alpha \beta}\right\rangle^{(\dot{\gamma})} / V$ in the non-equilibrium stationary state (neglecting the diagonal contribution that gives the pressure) is approximated assuming that stress relaxations can be computed from integrating the transient density correlations

$$
\sigma_{\alpha \beta}(\dot{\gamma})=\frac{n k_{B} T}{2} \int_{0}^{\infty} d t \int \frac{d^{2} q}{(2 \pi)^{2}} \frac{\partial S_{q(-t)}}{\partial t} \frac{q_{\alpha} q_{\beta}}{q} \frac{\partial c_{q}}{\partial q} \Phi_{\mathbf{q}(-t)}^{2}(t) .
$$

Flow also leads to the build up of shear-induced micro-structural changes, which, again integrating up the transient density correlators, can be found from

$$
S_{\mathbf{q}}(\dot{\gamma})=S_{q}+\int_{0}^{\infty} d t \frac{\partial S_{q(-t)}}{\partial t} \Phi_{\mathbf{q}(-t)}^{2}(t)
$$

A far smaller isotropic term in $S_{\mathbf{q}}(\dot{\gamma})$ (see (Fuchs Cates 2009)) is neglected here, as it is of importance for the plane perpendicular to the flow only.

\section{Numerical aspects}

The set of coupled Eqs. 3.13 .2 and 3.3 was solved self-consistently using modifications of standard algorithms and the $\mathrm{Ng}$-iteration-scheme ( $\mathrm{Ng} 2009)$. The functions were discretized on a 2D-Fourier-grid consisting of 101 grid points in either coordinate direction using a cutoff of $q D=30$. More explicitly the Fourier-grid was discretized as $q_{x, y} D=-30,-29.4, \ldots, 0, \ldots, 29.4,30$ with $D$ being the particle diameter. In order to enhance the accuracy, the advected direct correlation function $c_{q(t)}$ in Eq. 3.3 was calculated from the input structure factor once it has been advected beyond the Fourier-grid cutoff up to $q D=100$. For the time-integration of the convolution integral in Eq. 3.1 an initial time step of $10^{-9} D^{2} / D_{0}$ was used. The time-integration in the calculation of derived quantities Eqs. 3.4 and 3.5 was performed by dividing the integration interval into two sub-intervals, the first one containing times shorter, the other one times longer than $t^{*}=1 /(1000 \dot{\gamma}) D^{2} / D_{0}$. The integration was then carried out on the intrinsic quasi-logarithmic grid of the MCT equation solver for times $0 \leq t \leq t^{*}$ and on an equally-spaced linear time-grid with spacing $\Delta t=t^{*}$ for times $t>t^{*}$.

\section{Equilibrium and transient quantities}

The MCT-ITT approach uses structural equilibrium correlations as input, computes transient structural density correlators to encode the competition between flowinduced and Brownian motion, and calculates all stationary properties from timeintegrals over the transient fluctuations. In this section, the input and intermediate quantities of the theoretical calculations are presented and discussed. 


\section{(a) Equilibrium structure factor}

The equilibrium structure factor $S_{q}$ is the only input quantity to the MCT-ITT equations. It varies smoothly with density or temperature, but leads to the transition from a shear-thinning fluid to a yielding glassy state at a glass transition density $\varphi_{c}$. Figure 1 shows modified hyper-netted chain structure factors of monodisperse hard discs in $d=2$ from (Bayer et al. 2007) used in the MCT-ITT calculations. For comparison, the averaged structure factors obtained from the binary mixture simulation are shown also. In both cases, the value of the glass transition density $\varphi_{c}$ is included in the curves in Fig. 1, and only smooth changes in $S_{q}$ are noticeable. The short range order at the average particle distance, as measured in the primary peak of $S_{q}$, increases with densification. Differences in the structure between the mono- and the bidisperse system become appreciable beyond the primary peak, and especially beyond the second peak in $S_{q}$. Also the height of the primary peak in $S_{q}$ at $\varphi_{c}$ differs, indicating that the averaged structure factor in the bidisperse system does not well characterize the local structure and caging in the simpler system, and that a comparison with a bidisperse MCT-ITT calculation should be performed. As this is outside the present numerical reach, we proceed by comparing results for the characteristic wavevectors denoted $q_{1}$ to $q_{4}$ in Fig. 1. The angular dependence of the anisotropic structure will be explored along the special directions indicated in the insets.
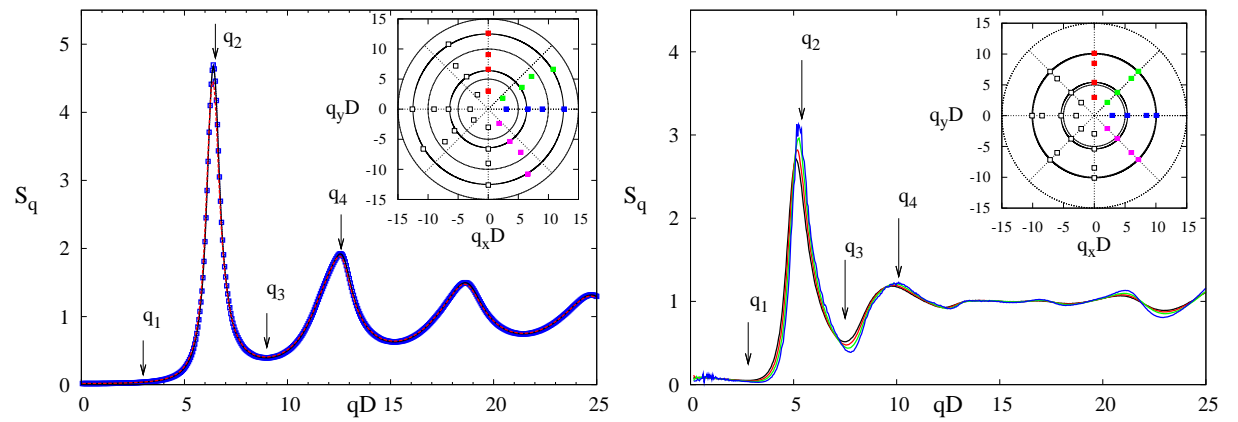

Figure 1. Left panel: modified hyper-netted chain structure factor, used as input quantity to MCT, for separation parameters $\varepsilon=-10^{-2}$ (red), $10^{-5}$ (black) and $10^{-3}$ (blue). The critical packing fraction is $\varphi_{c}=0.69899$. The selected wavevectors are $q_{1} D=3.0$, $q_{2} D=6.6, q_{3} D=9.0$ and $q_{4} D=12.6$. Right panel: averaged structure factors for the simulated binary system. The packing fractions are $\varphi=0.72,0.74,0.76$ and $\varphi=0.79$. The approximate critical packing fraction is $\varphi_{c} \simeq 0.79 \pm 0.005$, as estimated from the flow curves of Fig. 5 . Both insets exhibit the magnitude and the relative orientation of the selected wavevectors and introduce the colour code, which is used later on throughout the comparison. The wavevectors for the right panel are: $q_{1} D=2.8, q_{2} D=5.4, q_{3} D=7.5$ and $q_{4} D=10.1$.

\section{(b) Transient coherent density correlation functions}

The central quantities in MCT-ITT, which encode the competition between shear driven motion and random fluctuations, are the transient density correlators. Structural rearrangements manifest themselves as a (second) slow relaxation process 
in the $\Phi_{\mathbf{q}}(t)$, whose relaxation time depends sensitively on the distance to the glass transition, measured by the (relative) separation parameter $\varepsilon=\left(\varphi-\varphi_{c}\right) / \varphi_{c}$, and on the magnitude of the shear rate. Figure2 shows representative curves in a fluid state (left column) and in a shear-melted glassy state (right column) for the wavevectors and directions defined in Fig. 11, and for nine different shear rates. The denoted bare Peclet numbers measure the shear rate compared to the Brownian diffusion time estimated with the single particle diffusion coefficient $D_{0}$ at infinite dilution. Characteristically, for the present strongly viscoelastic system, shear affects the structural relaxation already at extremely small bare Peclet numbers $\mathrm{Pe}_{0} \ll 1$. The short time motion, which corresponds to the local diffusion of the particles within their neighbour cages, however, is not much affected as long as $\mathrm{Pe}_{0}<1$ holds.

In the fluid $(\varepsilon<0)$, a linear response regime, where shear does not affect the decay of thermal fluctuations, is observed for small (dressed) Peclet or Weissenberg numbers $\mathrm{Pe} \leq 1$, where $\mathrm{Pe}=\dot{\gamma} \tau$ measures the shear rate relative to the intrinsic relaxation time. The latter can be estimated from the relaxation of $\Phi_{q_{2}}(t)$, viz. at the primary peak of $S_{q}$, where the structure relaxes most slowly in the quiescent case. The final (or $\alpha$-) relaxation time $\tau$ increases strongly when approaching the glass transition; with $\tau \propto(-\varepsilon)^{2.38}$ predicted by MCT (Bayer et al. 2007). For the fluid state in Fig. 2, only $\mathrm{Pe}_{0} \leq 10^{-6}$ is small enough that $\mathrm{Pe}<1$ holds and the final relaxation curves agree for different shear rates. In the glass $(\varepsilon \geq 0)$, shear is the origin of the decay of the otherwise frozen-in density fluctuations, and all nine shear rates lead to different final decays. While for small and larger wavevectors the final decay is rather isotropic, around the main peak in $S_{q}$ some anisotropy is noticeable in the transient correlators. In the direction perpendicular to the flow, $q_{x}=0$ (red), shear is not as efficient in decorrelating the density as in the other directions, which fall closer together.

One of the central predictions of MCT-ITT concerns the existence of a scaling law describing the yielding of glassy states, which manifests itself by an approach to a master function $\Phi_{\mathbf{q}}(t) \rightarrow \tilde{\Phi}_{\mathbf{q}}(\tilde{t})$ for decreasing shear rate, $\dot{\gamma} \rightarrow 0$ and $\varepsilon \geq 0$, where the rescaled time $\tilde{t}=\dot{\gamma} t$ agrees with the accumulated strain. This scaling law, which is quite obvious in Fig. 2, is tested quantitatively for some wavevectors in Fig. 3 , and holds extremely well (for $q_{1}$ ), or with pre-asymptotic corrections (for $q_{2}$ ). The shapes of the yielding master functions can be very well fitted with compressed exponentials, $\Phi_{\mathbf{q}}(t) \simeq A_{\mathbf{q}} \exp \left(-\left(t / \tau_{\mathbf{q}}\right)^{\beta_{\mathbf{q}}}\right)$, but the parameters, including the exponent $\beta_{\mathbf{q}}$, depend on wavevector and orientation; see Table 1 for representative values.

The quite good fit to compressed exponentials is at present only a numerical observation. Yet, close to the glass transition asymptotic expansions are possible which analytically predict the initial part of the yield master functions (Fuchs Cates 2002,2003,2009):

$$
\left.\tilde{\Phi}_{\mathbf{q}}(\tilde{t} \rightarrow 0, \varepsilon=0) \rightarrow f_{q}^{c}-h_{q} \sqrt{c^{(\dot{\gamma})} /\left(\lambda-\frac{1}{2}\right)}\right) \tilde{t}+\ldots .
$$

As the critical glass form factor $f_{q}^{c}$ and the critical amplitude $h_{q}$ are isotropic, this result suggests a rather isotropic yielding process right at the glass transition density. Approximating $\tilde{\Phi}_{\mathbf{q}}(\tilde{t})$ by an exponential, it also provides an estimate for the final relaxation time under shear. Except for $c^{(\dot{\gamma})}$ all quantities in the above formula have been determined in quiescent MCT (Bayer et al. 2007), and our values 

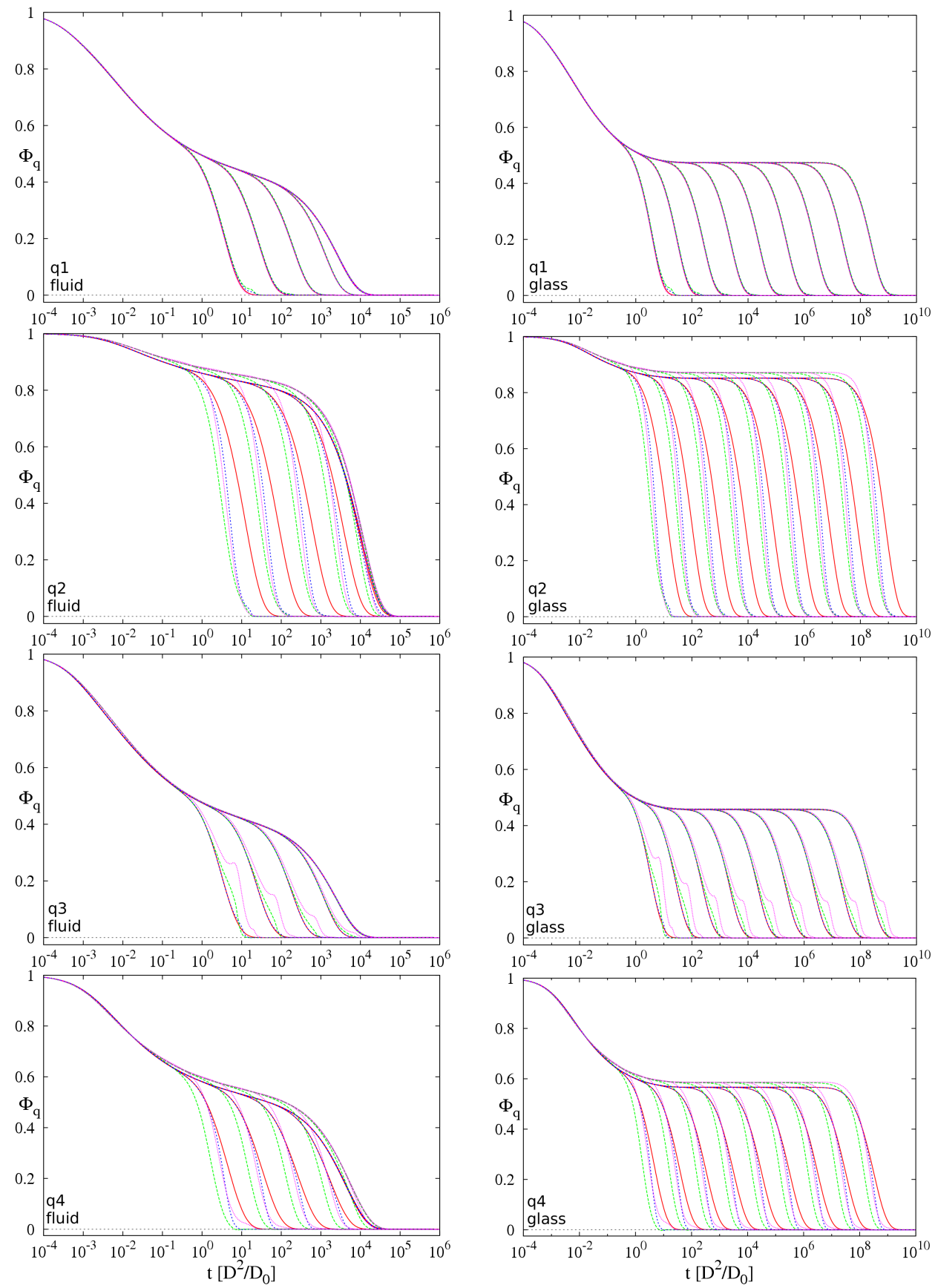

Figure 2. Transient density correlators in MCT in the liquid state (left column) for $\varepsilon=-10^{-3}$ and in the glassy state (right column) at $\varepsilon=10^{-3}$ at $q_{1}, q_{2}, q_{3}$ and $q_{4}$ (from top to bottom) and $\mathrm{Pe}_{0}=10^{-1} \ldots 10^{-9}$ (from left to right). The colour code gives the orientations according to Fig. 1 The difference of the plateau heights in the second and fourth row emerges as a discrepancy of the magnitudes and orientations of wavevectors due to the selected discretization. 

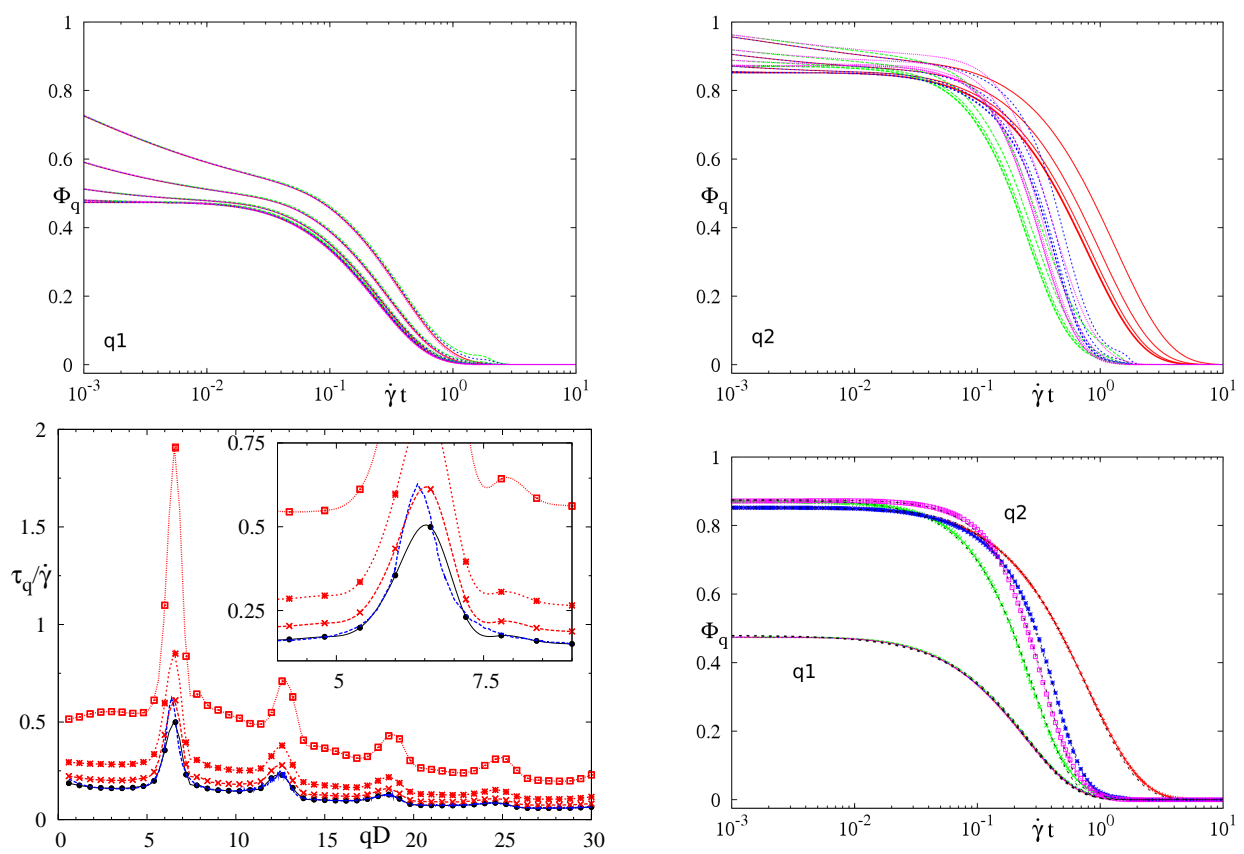

Figure 3. Top row: transient density correlators as functions of accumulated strain $\dot{\gamma} t$ in the glassy state at $\varepsilon=10^{-3}$ for the wavevectors $q_{1}$ (top left), $q_{2}$ (top right); collapse/ approach onto a master function appears for Peclet numbers ranging from $\mathrm{Pe}_{0}=10^{-1}$ to $10^{-9}$. Bottom right, master functions of this glassy state at $q_{1}$ (isotropic, lines) and $q_{2}$ (anisotropic, differently colored symbols and lines). The dashed black lines in this panel show compressed exponential fits $\Phi_{\mathbf{q}}(t) \simeq A_{\mathbf{q}} \exp \left(-\left(t / \tau_{\mathbf{q}}\right)^{\beta_{\mathbf{q}}}\right)$ with parameters according to Table 1 Bottom left: rescaled final relaxation times $\tau_{\mathbf{q}} / \dot{\gamma}$ for $q_{x}=0, \mathrm{Pe}_{0}=10^{-9}$ and $\varepsilon=10^{-2}, 10^{-3}$ and $10^{-4}$ (red from top to bottom) and $10^{-5}$ (black). The blue line gives the estimate deduced from the stability analysis $\tau_{\mathbf{q}} / \dot{\gamma} \simeq f_{q}^{c} /\left(h_{q} \sqrt{c^{(\dot{\gamma})} /\left(\lambda-\frac{1}{2}\right)}\right)$ for $c^{(\dot{\gamma})}=3.5$. The inset shows a close-up of the region around the first peak.

(e.g. $\lambda=0.698$ ) differ only because of the coarser discretization in $\mathbf{q}$ space that is necessary under shear. Close to the glass transition, we estimate $c^{(\dot{\gamma})} \approx 3.5$. As the bottom left panel in Fig. 3 and Fig. 2 both show, this estimate for the final relaxation time is quite good close to the transition, but deeper in the glass the correlators become somewhat more anisotropic. Specifically for $q_{x}=0$ (red) with wavevector magnitudes around $|\mathbf{q}| \simeq q_{2}$, the correlator slows down relative to other orientations.

More detailed analytical predictions, are possible around $f_{q}^{c}$, where spatial and temporal dependences in the transient fluctuations decouple (Fuchs Cates 2003):

$$
\Phi_{\mathbf{q}}(t)=f_{q}^{c}+h_{q} \sqrt{C|\varepsilon|} \mathcal{G}_{ \pm}\left(t / t_{\sigma},|\dot{\gamma}| t_{\sigma} \sqrt{c^{(\dot{\gamma})} / C|\varepsilon|}\right)+\ldots
$$

$\mathcal{G}_{ \pm}$is a universal function, which only depends on $\lambda$, and $t_{\sigma} \propto|\varepsilon|^{-1.56}$ is another MCT time scale that diverges at the glass transition (Bayer et al. 2007). This factorization property known from quiescent MCT generalizes to steady shear, and 


\begin{tabular}{||c|c|c|c|c|c|c||}
\hline $\mathbf{q}$ & $|\mathbf{q}| / D$ & $q_{x} / D$ & $q_{y} / D$ & $A_{\mathbf{q}}$ & $\beta_{\mathbf{q}}$ & $\tau_{\mathbf{q}} \cdot \dot{\gamma}$ \\
\hline \hline$q_{1}$ red & 3.0 & 0 & 3.0 & 0.479 & 1.121 & 0.255 \\
\hline$q_{1}$ green & 3.0 & 2.4 & 1.8 & 0.481 & 1.117 & 0.263 \\
\hline$q_{1}$ blue & 3.0 & 3.0 & 0 & 0.479 & 1.112 & 0.258 \\
\hline$q_{1}$ magenta & 3.0 & 1.8 & -2.4 & 0.480 & 1.117 & 0.249 \\
\hline$q_{2}$ red & 6.6 & 0 & 6.6 & 0.856 & 1.084 & 0.811 \\
\hline$q_{2}$ green & 6.5 & 5.4 & 3.6 & 0.878 & 1.384 & 0.291 \\
\hline$q_{2}$ blue & 6.6 & 6.6 & 0 & 0.875 & 1.670 & 0.363 \\
\hline$q_{2}$ magenta & 6.5 & 3.6 & -5.4 & 0.849 & 1.617 & 0.431 \\
\hline \hline
\end{tabular}

Table 1. Parameters of the compressed exponentials $A_{\mathbf{q}} \exp \left(-\left(t / \tau_{\mathbf{q}}\right)^{\beta_{\mathbf{q}}}\right)$ fitted to the yield master functions shown in Fig. 3 .
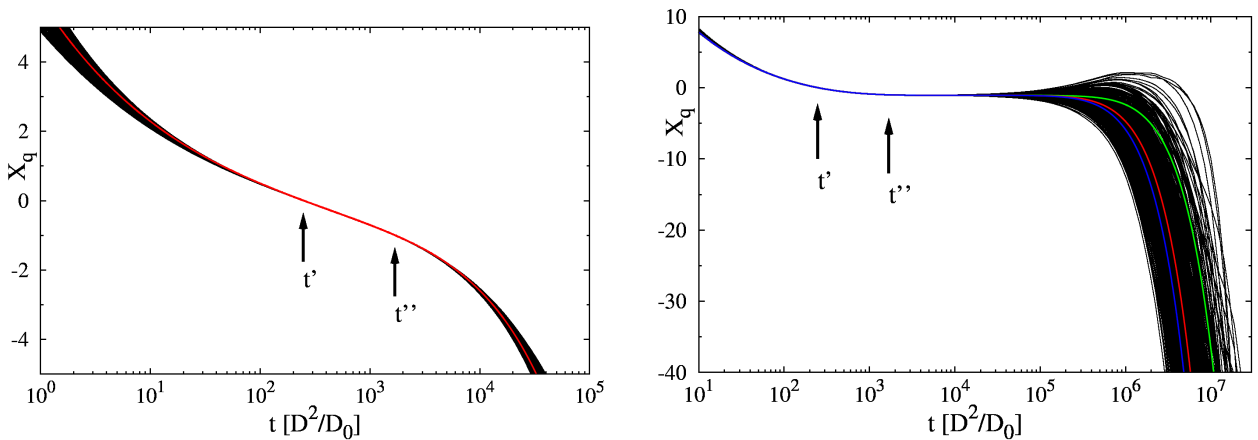

Figure 4. Factorization property: $X_{\mathbf{q}}(t)=\frac{\Phi_{\mathbf{q}}(t)-\Phi_{\mathbf{q}}\left(t^{\prime}\right)}{\Phi_{\mathbf{q}}\left(t^{\prime}\right)-\Phi_{\mathbf{q}}\left(t^{\prime \prime}\right)}$. The left picture shows the liquid state at $\varepsilon=-10^{-4}, \dot{\gamma}=10^{-8}$, while the right picture depicts the glassy state at $\varepsilon=10^{-4}, \dot{\gamma}=10^{-8}$. Black curves give the solutions of the MCT equations for $0 \leq q D \leq 24$. At the times $t^{\prime}=247$ and $t^{\prime \prime}=1683$ the correlators are approximately 0.01 above, respectively below their critical plateaus. Coloured curves are rescaled solutions of the modified $\beta$-scaling equation $X(t)=\frac{\mathcal{G}\left(t / t_{\sigma}\right)-\mathcal{G}\left(t^{\prime} / t_{\sigma}\right)}{\mathcal{G}\left(t^{\prime} / t_{\sigma}\right)-\mathcal{G}\left(t^{\prime \prime} / t_{\sigma}\right)}$ with $t_{\sigma}=965$ and correspond to values $c^{(\dot{\gamma})}=1$ (green), 3.5 (red) and 5 (blue). The coefficient $c^{(\dot{\gamma})}=3.5$ was also found in Fig. 3 and captures the shear driven decay of the glass structure.

is an essential feature of the localization transition that underlies glass formation in MCT. In Fig. 4, the quantity $X_{\mathbf{q}}(t)=\frac{\Phi_{\mathbf{q}}(t)-\Phi_{\mathbf{q}}\left(t^{\prime}\right)}{\Phi_{\mathbf{q}}\left(t^{\prime}\right)-\Phi_{\mathbf{q}}\left(t^{\prime \prime}\right)}$ is plotted which should become wavevector and orientation independent if factorisation holds. This holds in the liquid, and in the glass, where however shear leads to strong (anisotropic) pre-asymptotic corrections already for $\dot{\gamma} t \geq 0.01$.

\section{Results and comparisons}

Based on the approximated generalized Green-Kubo relations of Eqs. (3.4) and (3.5), and the properties of the transient correlators discussed in the previous section, MCT-ITT makes a number of predictions for stationary stresses and structural correlations (Fuchs Cates 2002, 2009). In the following, we will test these qualitatively, but also quantitatively, by comparing them to the two-dimensional simulation data. 


\section{(a) Stationary stresses}

The quantity of most interest in nonlinear rheology is the shear stress $\sigma_{x y}(\dot{\gamma})$. Flow curves giving the shear stress as function of the shear rate can be obtained in the simulations and are shown in Fig. 5. The viscosity decreases below its Newtonian value $\eta_{0}=\sigma_{x y}(\dot{\gamma} \rightarrow 0) / \dot{\gamma}$ upon increasing the shear rate already for small $\mathrm{Pe}_{0}$ values. Shear thinning in which the stress increases less than linearly with $\dot{\gamma}$, sets in at $\mathrm{Pe}$ of the order of unity, and this crossover shifts to lower and lower $\mathrm{Pe}_{0}$ for increasing density. At the density around $\varphi_{c} \approx 0.79$, the crossover leaves the accessible shear rate window. We use this as estimate of an ideal glass transition density, where the final relaxation time and the quiescent Newtonian viscosity $\left(\eta_{0} \propto\right.$ $\tau)$ diverge. Moreover, at this density the flow curves change from a characteristic $\mathrm{S}$ shape in the fluid, to exhibiting a rather $\dot{\gamma}$-independent plateau at small shear rates. This change is the hallmark of the transition in MCT-ITT between a shear thinning fluid and a yielding glass (Siebenbürger et al. 2009).

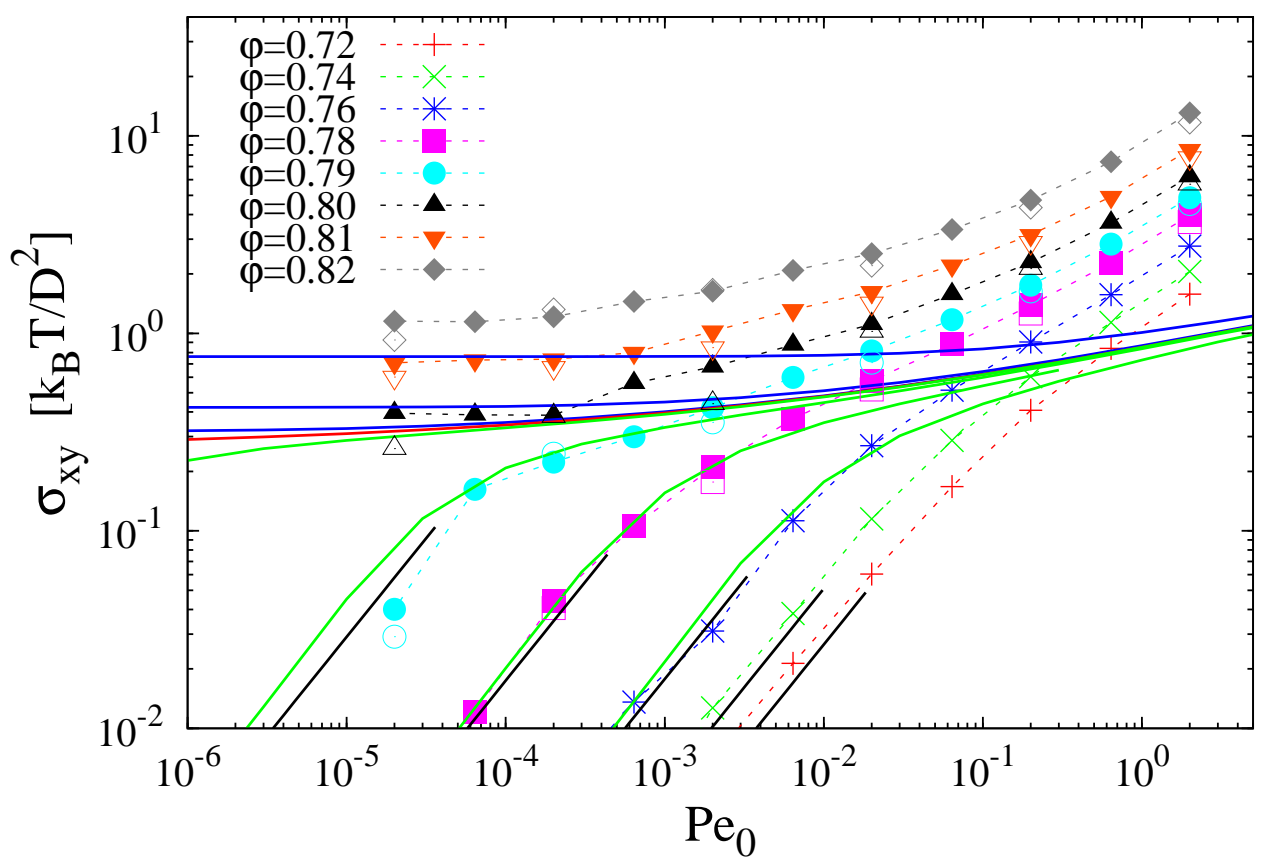

Figure 5. Shear stress $\sigma_{x y}(\dot{\gamma})$ versus shear rate given as Peclet number $\mathrm{Pe}_{0}=\dot{\gamma} D^{2} / D_{0}$ in MCT-ITT and simulation: The data points give simulation results for the densities denoted in the legend. Empty symbols show the results obtained via (2.4) and filled symbols the results from (2.3). The $\mathrm{Pe}_{0} \rightarrow 0$ (black solid lines) were calculated according to (2.5). The solid lines show calculations in MCT-ITT for $\varepsilon=10^{-2}, 10^{-3}, 10^{-4}, 10^{-5},-10^{-4},-10^{-3}$ and $-10^{-2}$ (from top to bottom; blue $\varepsilon>0$, red $\varepsilon \approx 0$, green $\varepsilon<0$ ). MCT-ITT results are shifted downwards by a factor 0.1 to match the simulation results.

The numerical MCT-ITT solutions show the same transition scenario, but because of the approximations involved, exhibit a different critical density than the simulations. Even if theory and simulation considered the same (binary) system, 


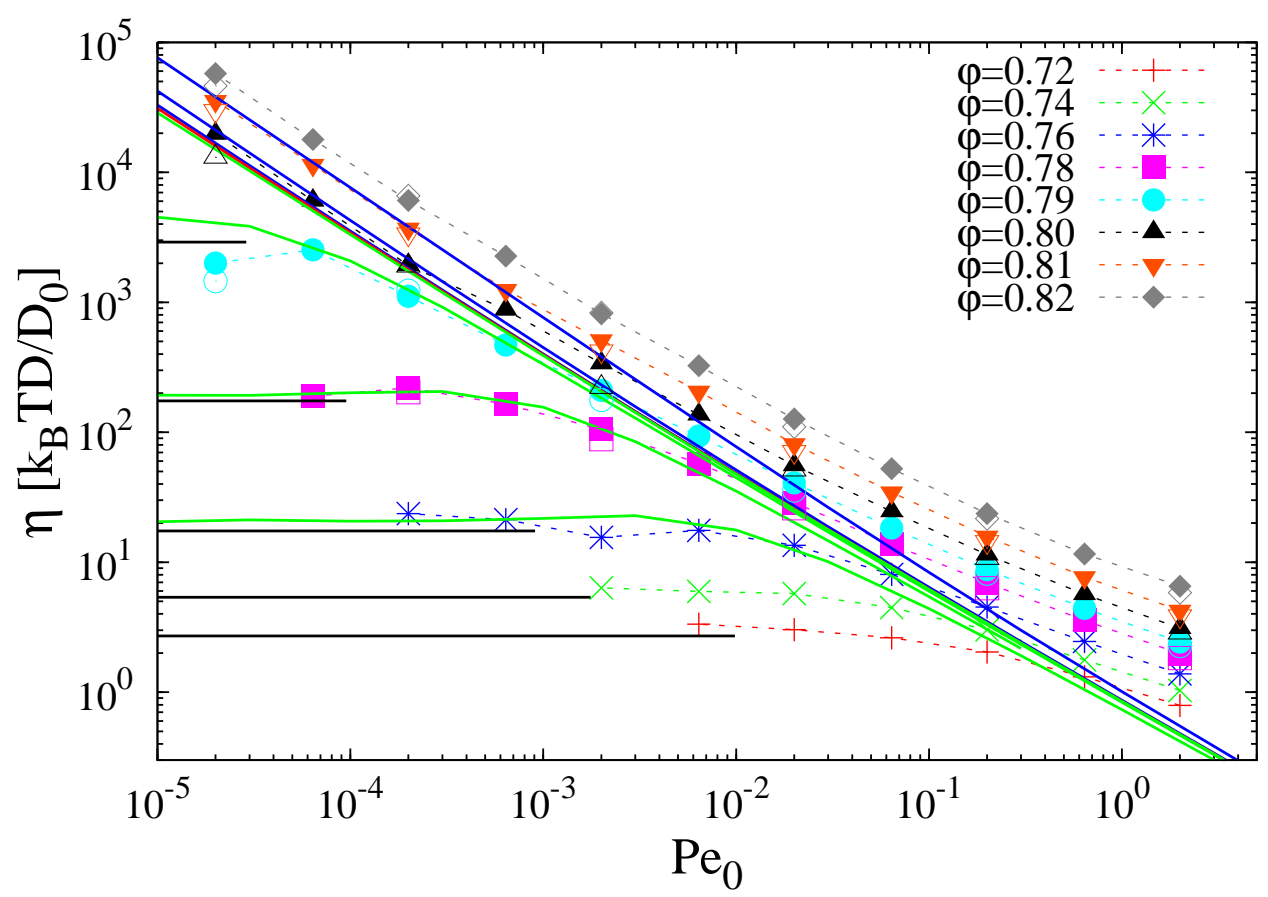

Figure 6. Viscosity $\eta(\dot{\gamma})$ versus shear rate given as Peclet number $\mathrm{Pe}_{0}=\dot{\gamma} D^{2} / D_{0}$ in MCT-ITT and simulation. The color codes are the same as in Figure 5 Again the empty symbols show the results of (2.4) and filled symbols the results from (2.3) while the $\mathrm{Pe}_{0} \rightarrow 0$ (black solid lines) were calculated according to 2.5. MCT-ITT results are shifted downwards by a factor 0.1 to match the simulation results.

a difference in critical packing fraction would be expected as is well known also in three dimensions (Voigtmann et al. 2004). Thus the following MCT-ITT calculations match the relative separation $\varepsilon$ from $\varphi_{c}$ in order to compare with the simulations. As MCT-ITT is aimed at describing the long time structural motion, errors need to be anticipated in its description of short time properties. This is obvious in real dispersions, where hydrodynamic interactions (neglected in MCTITT) affect the short time diffusion coefficient $D_{s}$, but may arise in the following comparisons, also. A rescaling of the effective Peclet number $\mathrm{Pe}_{0}^{\text {eff }}=\dot{\gamma} D^{2} / D_{s}$ would correct for this change in $D_{s}$. In order not to introduce additional fit parameters we refrain from doing so, but anticipate that future comparisons may require $D_{s} \neq D_{0}$.

The approach to a yield scaling law, where the final decay of the transient correlators depends on the accumulated strain only (see Figs. 2 and 3), predicts the existence of a (dynamical) yield stress $\sigma_{x y}^{+}(\varepsilon)=\sigma_{x y}(\dot{\gamma} \rightarrow 0, \varepsilon)$, which characterizes the shear melted glass. In the bidisperse hard disc mixture, at the glass transition it takes the (critical) value $\sigma_{x y}^{+, c}=\sigma_{x y}^{+}(\varepsilon=0) \approx 0.3 k_{B} T / D^{2}$; below the glass transition, the yield stress jumps to zero, $\sigma_{\alpha \beta}^{+}(\varepsilon<0)=0$. Its quantitative prediction is quite a challenge for theory, because Eq. 3.4 shows that it requires an accurate calculation of the shear driven relaxation process. MCT-ITT overestimates the critical yield stress $\sigma_{x y}^{+, c}$ by roughly a factor 10 because, presumably, the decay of the transient correlators is too slow. Yet, of course the difference between the monodisperse system in the MCT-ITT calculation and the bidisperse simulated 
system contributes in unknown way to the error. It appears reasonable to assume that mixing two species reduces the stresses under flow, which would explain part of the deviation.

At larger shear rates, the flow curves from simulation appear to approach a second Newtonian plateau which, presumably, strongly depends on the hard-core character of excluded volume interactions and is outside the reach of the present MCT-ITT. The latter, by using its sole input $S_{q}$ rather than pair potential, is not directly aware of hard-core constraints. We checked, however, that the states remain homogeneous and random up to the $\mathrm{Pe}_{0}$ values shown.
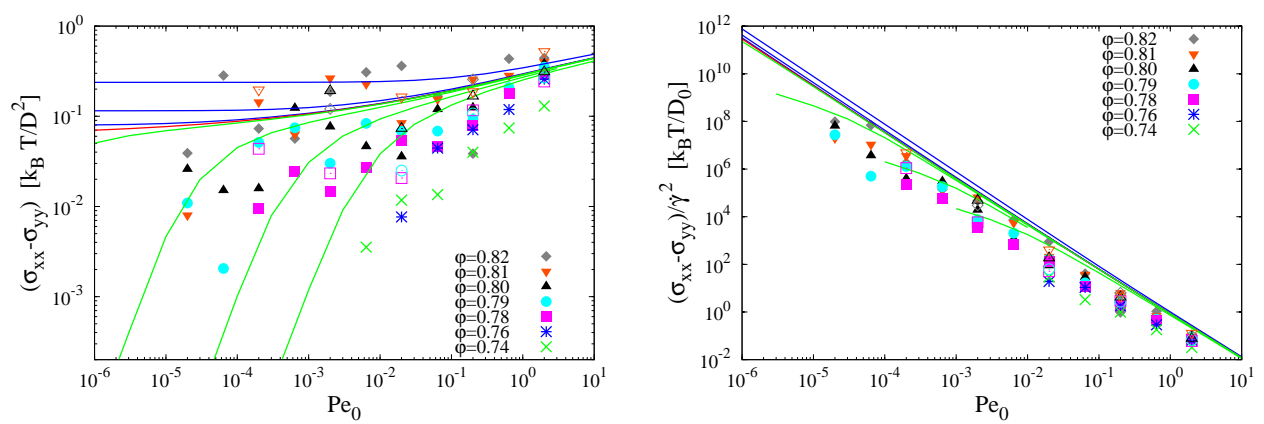

Figure 7. Left panel: Normal stress differences for the system at the densities given in the legend. The lines show MCT-ITT results at separation parameters $\varepsilon=10^{-2}, 10^{-3}, 10^{-4}, 10^{-5},-10^{-4},-10^{-3}$ and $-10^{-2}$ (from top to bottom) scaled by a factor 0.1 to match the simulation results. Right panel: Normal stress differences for the system divided by $\mathrm{Pe}_{0}^{2}$. Color codes and scaling factors are the same as in the left panel. In both panels empty symbols show the results obtained via (2.4) while filled symbols were calculated with (2.3).

Reassuringly, the same rescaling factor of 0.1 as for the shear stress brings theoretical and simulational normal stress differences, $\sigma_{x x}-\sigma_{y y}$, into register also; see Fig. 7 The normal stress differences are positive (indicating that the dispersion would swell after flowing through a nozzle), and show similar behavior to the stress, increasing like $\dot{\gamma}^{2}$ in the fluid for small shear rates, and leveling out onto a plateau in the yielding glass.

\section{(b) Distorted micro-structure}

The macroscopic stresses in the flowing dispersion are experimentally most important, but provide only an averaged description of the local effects of shear. Spatially resolved information can be learned from the distorted structure factor $\delta S_{\mathbf{q}}(\dot{\gamma})=S_{\mathbf{q}}(\dot{\gamma})-S_{q}$, which in MCT-ITT is connected to the stress via

$$
\sigma_{i j}=\frac{n k_{B} T}{2} \int \frac{d^{2} q}{(2 \pi)^{2}} \frac{q_{i} q_{j}}{q} \frac{\partial c_{q}}{\partial q} \delta S_{\mathbf{q}}(\dot{\gamma}),
$$

as follows from Eqs. (3.4) and (3.5).

Figure 8 shows colour-coded structure factors $S_{\mathbf{q}}(\dot{\gamma})$ as function of the twodimensional wavevector $\mathbf{q}$, with $q_{x}$ in the direction of flow and $q_{y}$ along the gradient direction. In the left column, panels with simulation data are compared to panels 

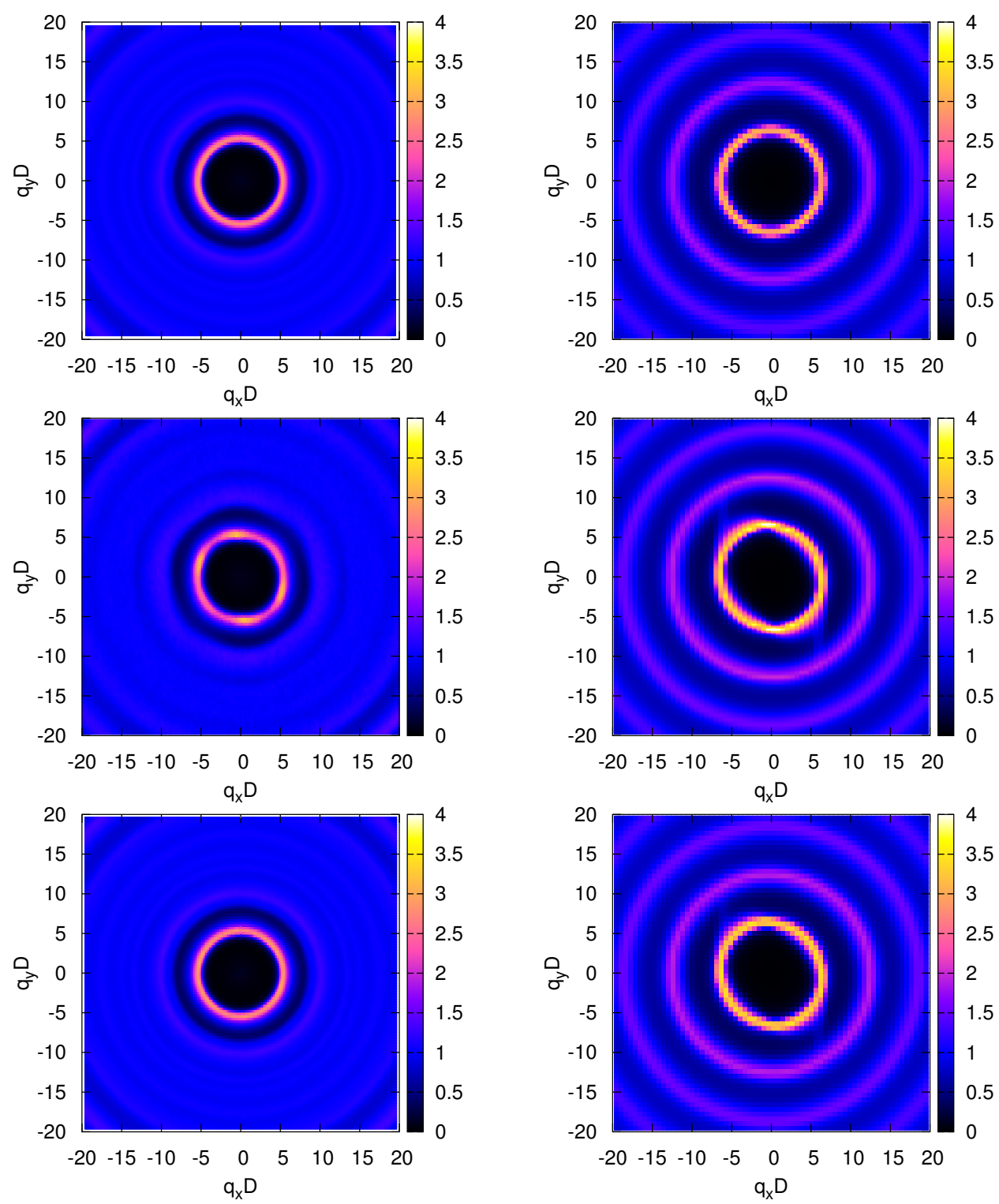

Figure 8. Distorted structure factor $S(\mathbf{q}, \dot{\gamma})$ from simulation (left column) and from theory (right column): for states in linear response regime in the liquid (top row: simulation $\varphi=0.78$ and $\mathrm{Pe}_{0}=2 \cdot 10^{-4} \mathrm{MCT} \varepsilon=-10^{-2}$ and $\left.\mathrm{Pe}_{0}=10^{-4}\right)$; in the glass at high shear rate (middle row: simulation $\varphi=0.79$ and $\mathrm{Pe}_{0}=2$, MCT $\varepsilon=10^{-3}$ and $\mathrm{Pe}_{0}=10^{-2}$ ); and in the glass at small shear rate (bottom row: simulation $\varphi=0.79$ and $\mathrm{Pe}_{0}=2 \cdot 10^{-4}$, $\operatorname{MCT} \varepsilon=10^{-3}$ and $\left.\mathrm{Pe}_{0}=10^{-4}\right)$.

in the right column obtained in MCT-ITT. Scattering intensities are presented (i) (top row) in the linear response regime in the fluid, effectively measuring the equilibrium structure factors already shown in Fig. 1, (ii) (middle row) at high shear in the glass, where all densities are in the shear thinning region, and (iii) (bottom row) in the glass at low shear rate, where the yielding glassy state is 
tested. While the fluid $S_{\mathbf{q}}(\dot{\gamma})$ is isotropic for small $\mathrm{Pe}_{0}$ (case $(i)$ ), as required by linear response theory, increasing $\mathrm{Pe}_{0}$ to values around unity, leads to an ellipsoidal scattering ring, which is elongated along the so-called 'compressional axis' $q_{x}=$ $-q_{y}$, and more narrow along the 'extensional axis' $q_{x}=q_{y}$; this indicates that shear pushes particles together along the compressional and pulls particles apart along the extensional diagonals (Vermant Solomon 2005). Theory and simulation data in this representation qualitatively agree except for that MCT-ITT somewhat overestimates the anisotropic distortion of the glass structure at low $\mathrm{Pe}_{0}$.

A more careful look onto the distorted microstructure is provided by $q$-dependent cuts through $S_{\mathbf{q}}(\dot{\gamma})$ along the directions colour-coded in Fig. 1. Important for the direction $q_{x}=0$ (red) is the need, present in both simulation and theory, to average $S_{\mathbf{q}}(\dot{\gamma})$ over a small but finite angle, because exactly at $q_{x}=0$ the structure oscillates noisily around zero.

Especially of interest are the intensities of case ( iii), where the stationary structure of the shear melted solid is studied. The density $\varphi=0.79$ is not yet high enough to lie in the glass, but close enough to the glass transition so that the correlations at quite low rate, namely $\mathrm{Pe}_{0}=210^{-4}$, closely resemble the ones of glassy states at very low shear rates. (While we can get good statistics for stresses at $\mathrm{Pe}_{0}=210^{-5}$ for all densities, structure factors can not be sampled sufficiently there.) At this density of $\varphi=0.79$ also the equilibrium structure factor $S_{q}$ can be obtained in long simulation runs, and the (relative) difference $\left(S_{\mathbf{q}}(\dot{\gamma}, \varepsilon)-S_{q}\right) / S_{q}$ can thus be determined. It is shown in Fig. 9 for two states in order to investigate the distorted structure of the shear melted glass in detail. We base the following discussion on the hypothesis that $\mathrm{Pe}_{0}=2 \cdot 10^{-4}$ at $\varphi=0.79$ captures a glass-like state in the limit of low bare Peclet number.

Figure 9 provides a sensitive test of the accuracy of the theoretical predictions. The lower left panel shows that the structure factor at vanishing shear rate $S_{\mathbf{q}}(\dot{\gamma} \rightarrow$ $0)$ jumps discontinuously at the glass transition; while $S_{\mathbf{q}}(\dot{\gamma} \rightarrow 0) \rightarrow S_{q}$ holds in the fluid, $S_{\mathbf{q}}(\dot{\gamma} \rightarrow 0) \neq S_{q}$ holds in the glass. Relative deviations $\left(S_{\mathbf{q}}(\dot{\gamma} \rightarrow 0)-S_{q}\right) / S_{q}$ of $20 \%$ remain. Simulation finds quite isotropic deviations which show a maximum on the low- $q$ side of the primary peak in $S_{q}$. MCT-ITT predicts the absence of a linear response regime in $S_{\mathbf{q}}(\dot{\gamma})$ as function of the shear rate in the glass, and derives it from the existence of the yield scaling law in the transient correlators. Because $\dot{\gamma}$ sets the time scale for the final relaxation into the stationary state, the limit $\dot{\gamma} \rightarrow 0$ does not agree with the quiescent result $\dot{\gamma}=0$.

Quantitatively, MCT-ITT overestimates the distortion again by a factor up to 10 , and finds a noticeable anisotropy, as discussed in context with Fig. 8. While the difference between the bidisperse and the monodisperse system may influence the comparison, we believe that the major origin of the error is that MCT-ITT underestimates the speeding up of structural rearrangements caused by shear. The too slow transient correlators thus become anisotropic because the accumulated strain $\dot{\gamma} t$ becomes too big before structural correlations have decayed.

Qualitatively, aspects of the anisotropy predicted by MCT-ITT can be seen in the simulations at only slightly larger shear rates, like at $\mathrm{Pe}_{0}=0.2$ shown in the middle left panel of Fig. 9. While along the two axis- and the extensional diagonal direction, the low- $q$ wing of the primary peak in $S_{\mathbf{q}}(\dot{\gamma})$ becomes enhanced under shear, along the compressional axis (magenta) it gets suppressed, and the high- $q$ wing is pushed up. Simulation also finds a suppression of the peak height along all 

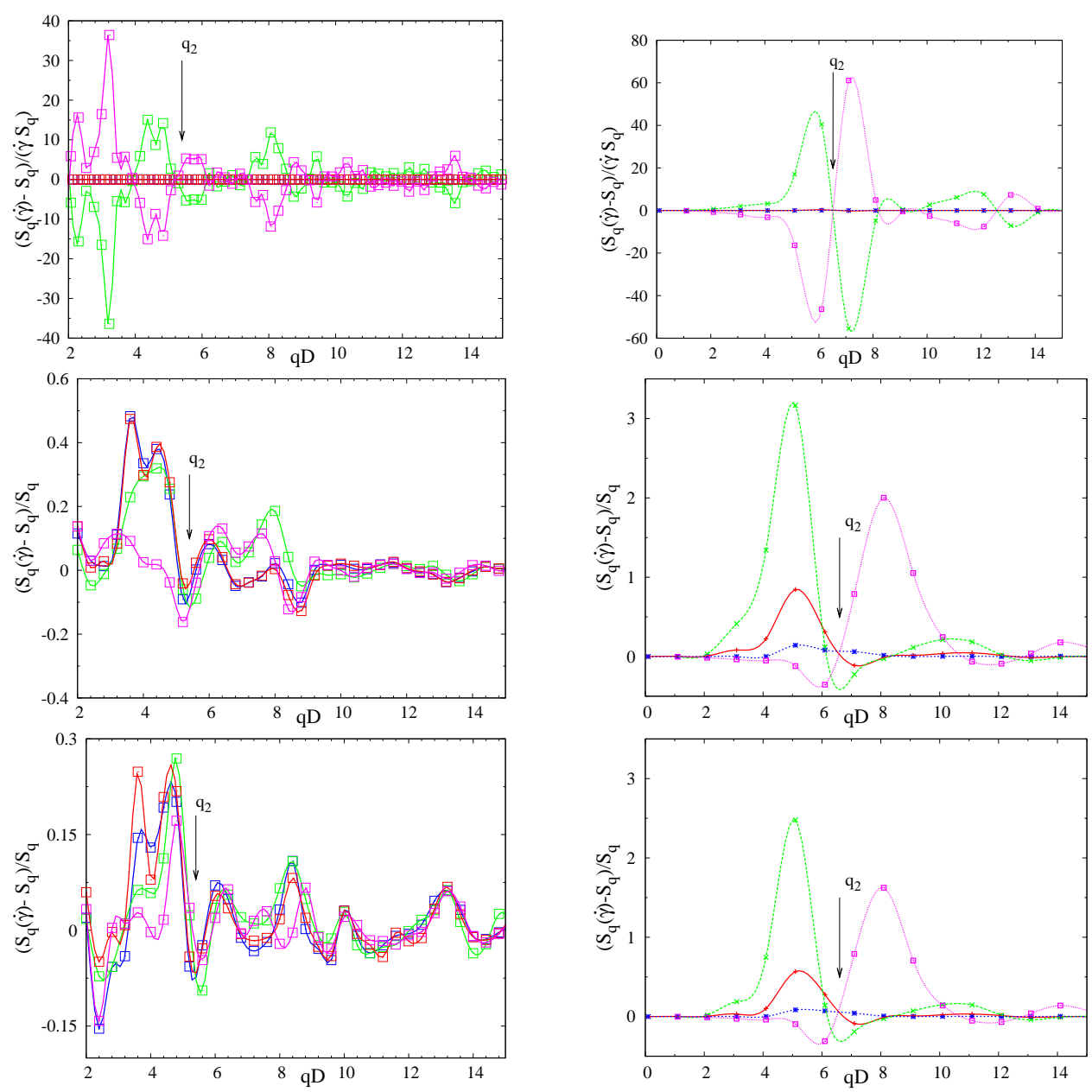

Figure 9. Direct comparison of the quantity $\left(S(\mathbf{q}, \dot{\gamma})-S_{q}\right) / S_{q}$ between simulation and MCT. The selected orientations are: $q_{x}=0$ (red), $q_{x}=q_{y}$ (green), $q_{y}=0$ (blue) and $q_{x}=-q_{y}$ (magenta) for $\varphi=0.79$ in the simulation (left column). The $\mathrm{Pe}_{0}$ numbers are $4 \cdot 10^{-4}$ (top row), $2 \cdot 10^{-1}$ (middle row) and $2 \cdot 10^{-3}$ (bottom row). For MCT (right column) the values are: $\varepsilon=-10^{-2}, \mathrm{Pe}_{0}=10^{-4}$ (top row), $\varepsilon=10^{-3}, \mathrm{Pe}_{0}=10^{-2}$ (middle row) and $\varepsilon=10^{-3}, \mathrm{Pe}_{0}=10^{-8}$ (bottom row). Note that in the linear response case (top row) results were normalized by the shear rate $\dot{\gamma}$. All structure factors are averaged over a small angle in the $\mathbf{q}$ direction (for an absolute $|\boldsymbol{q}|$-value and an angle $\varphi$ a small section $\Delta q$ and $\Delta \varphi$ is defined; all $\boldsymbol{q}$ values lying within the defined section are used for the averaging. $\left.\Delta \varphi_{\mathrm{sim}}=5^{\circ}, \Delta\left|\boldsymbol{q}_{\mathrm{sim}}\right|=0.4, \Delta \varphi_{\mathrm{MCT}}=15^{\circ}, \Delta\left|\boldsymbol{q}_{\mathrm{MCT}}\right|=1.0.\right)$.

directions, which MCT-ITT reproduces along the diagonal directions. Overall the anisotropy and the magnitude of the distortions predicted by MCT-ITT remain too large, but the deviation decreases. The differences between the equilibrium structure factors $S_{q}$ in the simulated and in the calculated system should be taken into account in future work, and presently preclude comparisons at larger wavevectors. 


\section{Conclusions and Outlook}

The first fully quantitative solutions of the MCT-ITT equations for the distorted microstructure and the stresses in steadily sheared two-dimensional shear-thinning fluids and yielding glasses of Brownian hard discs exhibit all the universal features discussed within schematic MCT-ITT models (Fuchs Cates 2003). They compare qualitatively well, but quantitatively with appreciable errors, with Brownian dynamics simulations of a bidisperse mixture without hydrodynamic interactions in a linear shear profile, which for all states considered remains in an homogeneous and disordered state. The non-analytic behavior of the stationary properties, and the lack of a linear response regime throughout the (shear-melted) glass state, predicted by theory, can be found in the simulation.

\section{Acknowledgements}

Work funded in part by DFG-IRTG 667, and EPSRC grants EP/045316 and EP/030173. MEC holds a Royal Society Research Professorship

\section{References}

Alder B. J., Gass D. M. Wainwright T. E. 1970 J. Chem. Phys. 533813.

Bayer, M., Brader, F. Ebert, E. Lange, M. Fuchs, G. Maret, R. Schilling, M. Sperl, Wittmer, J. P. 2007 Phys. Rev. E 76011508.

Brader, J., Cates, M. E Fuchs, M. 2008 Phys. Rev. Lett. 101138301.

Foss, D. R. Brady, J. F. 2000 J. Rheol 44 629-651.

Fuchs, M. Cates, M. E. 2002 Phys. Rev. Lett. 89248304.

Fuchs, M. Cates, M. E. 2002 Faraday Discuss. 123 267-286.

Fuchs, M. Cates, M. E. 2009 , J. Rheol. 53(4) 957-1000.

Furukawa, A., Kim, K., Saito, S. Tanaka, H. 2009 Phys. Rev. Lett. 102016001.

Lange, E., Caballero, J. B., Puertas, A. M. Fuchs, M. 2008 J. Chem. Phys. 130174903

Miyazaki K., Reichmann D. R. Yamamoto, R. 2004 Phys. Rev. E 70011501.

Ng, K.-C. 1974 J. Chem. Phys. 61 2680-2689.

Petekidis, G., Moussaï, A. Pusey, P. N. 2002 Phys. Rev. E 66051402.

Scala, A., Voigtmann, T. De Michele, C. 2007 J. Chem. Phys. 126134109.

Strating, P. 1999 Phys. Rev. E 592175.

Siebenbürger, M., Fuchs, M., Winter, H. H. Ballauff, M. 2009 J. Rheol 53 707-726.

Vermant, J. Solomon, M. J. 2005 J. Phys.: Condens. Matter 17 R187-R216

Voigtmann, Th., Puertas, A. M. M. Fuchs 2004 Phys. Rev. E 70061506.

Yamamoto, R. Onuki, A. 1998 Phys. Rev. E 58 3515-3529. 